\title{
A MSLN-targeted multifunctional nanoimmunoliposome for MRI and targeting therapy in pancreatic cancer
}

This article was published in the following Dove Press journal:

International Journal of Nanomedicine

18 September 2012

Number of times this article has been viewed

\section{Li Dengl,\# \\ Xingfa $\mathrm{Ke}^{4, \#}$ \\ Zhiying $\mathrm{He}^{3, \#}$ \\ Daoqiu Yang ${ }^{5}$ \\ Hai Gong ${ }^{6}$ \\ Yingying Zhang' \\ Xiaolong Jing ${ }^{4}$ \\ Jianzhong $\mathrm{YaO}^{2}$ \\ Jianming Chen'}

'Department of Pharmaceutics,

2Department of Medicinal Chemistry, School of Pharmacy, ${ }^{3}$ Department of

Cell Biology, Second Military Medical University, Shanghai, People's Republic of China; ${ }^{4}$ Department of Pharmacy, Fujian University of Traditional Chinese Medicine, Fujian, People's Republic of China; ${ }^{5}$ Department of Dermatology, 107th Hospital of PLA, Yantai, People's Republic of China; ${ }^{6}$ Department of Radiation Oncology, General Hospital of Jinan Military Region, Jinan, People's Republic of China

\#These authors contributed equally to this work

Correspondence: Jianming Chen

Department of Pharmaceutics, School of Pharmacy, Second Military Medical University, 325 Guohe Road, Shanghai, 200433, People's Republic of China

$\mathrm{Tel}+862|8| 87$ |29|

Fax +86 21 8I87 129|

Email yjcjm@।63.com

Correspondence: Jianzhong Yao Department of Medicinal Chemistry, School of Pharmacy, Second Military Medical University, 325 Guohe Road, Shanghai, 200433, People's Republic of China

Tel +862181871235

Fax +86 218187 I235

Email yaojz@shl63.net
Abstract: Pancreatic cancer is a highly lethal disease with a 5-year survival rate less than 5\% due to the lack of an early diagnosis method and effective therapy. To provide a novel early diagnostic method and targeted therapy for pancreatic cancer, a multifunctional nanoimmunoliposome with high loading of ultrasmall superparamagnetic iron oxides (USPIOs) and doxorubicin (DOX) was prepared by transient binding and reverse-phase evaporation method, and was conjugated with anti-mesothelin monoclonal antibody by post-insertion method to target anti-mesothelin-overexpressed pancreatic cancer cells. The in vitro and in vivo properties of this anti-mesothelin antibody-conjugated PEGlyated liposomal DOX and USPIOs (M-PLDU; and PEGlyated nanoimmunoliposome without antibody conjugation [PLDU]) were evaluated both in human pancreatic cancer cell line Panc-1 cell and in a pancreatic cancer xenograft animal model. Results showed that M-PLDUs were spherical and uniform with a diameter about $\sim 180 \mathrm{~nm}$, with a zeta potential of about $-28 \sim-30 \mathrm{mV}$, and had good efficacy encapsulating DOX and USPIOs. The in vitro study demonstrated that M-PLDUs possessed good magnetic resonance imaging (MRI) capability with a transverse relaxivity $\left(\mathrm{r}^{2}\right)$ of about $58.5 \mathrm{mM}^{-1} \cdot \mathrm{s}^{-1}$. Confocal microscopy showed more efficient uptake of M-PLDU in Panc-1 cells by antibodymediated targeting. Methyl thiazolyl tetrazolium assay results showed significant inhibitory effect of M-PLDU against Panc-1 cells (half-maximal inhibitory concentration, $1.95 \mu \mathrm{M}$ ). The in vivo imaging study showed that the tumor signal intensity (SI) dropped significantly about 4 hours after intravenous injection of M-PLDU. The decrease in tumor SI induced by M-PLDUs $(\Delta \mathrm{SI}=145.98 \pm 20.45)$ or PLDUs $(\Delta \mathrm{SI}=75.69 \pm 14.53)$ was much more significant than that by free USPIOs $(\Delta \mathrm{SI}=42.78 \pm 22.12 ; P<0.01)$. The in vivo antitumor study demonstrated that compared with FD (free DOX) and PLDU, M-PLDU possessed higher inhibitory effect on tumor growth and the tissue distribution assay further proved that M-PLDUs could selectively accumulate in the tumor xenograft. These results indicated that M-PLDU not only well retained the inherent MRI capability of USPIOs, but significantly improved the targeting distribution of USPIOs and therapeutic agents in pancreatic tumor tissues. They may serve as a promising theranostic nanomedicine not only for early detection but also for MRI-monitored targeting therapy of human pancreatic cancer.

Keywords: theranostic nanomedicine, nanoimmunoliposome, mesothelin, USPIO, doxorubicin, pancreatic cancer

\section{Introduction}

Pancreatic cancer is a highly lethal cancer. Despite significant advances in the treatment of many other human tumors, the 5-year survival rate for patients diagnosed with pancreatic cancer has not changed in decades and remains $<5 \%$. ${ }^{1}$ The high mortality rate of pancreatic cancer is mainly due to the difficulty in obtaining an early diagnosis 
and the lack of effective therapeutic strategies. The existing chemotherapeutic strategies including drugs such as 5-fluorouracil and gemcitabine, which have many off-target side effects, have shown disappointing results. ${ }^{2}$ Therefore, there is an urgent need to develop a technique for earlier diagnosis and a novel targeting therapeutic strategy.

Mesothelin (MSLN) is a differentiative antigen that is highly expressed in several human tumors, including mesothelioma, ovarian, and pancreatic cancers. ${ }^{3,4}$ As MSLN is overexpressed only in tumor tissues and not in adjacent normal tissues in pancreatic cancer, and is closely associated with tumor progression and migration, it has been proven to be a promising cancer biomarker and an effective target for cancer-targeted therapy. ${ }^{5,6}$ Modification with an anti-MSLN antibody would improve the targeting efficiency of diagnostic imaging agents and the drug-delivery system towards the MSLN-overexpressing pancreatic tumors.

Theranostic nanomedicine ${ }^{7}$ integrates cell targeting, ultrasensitive imaging and therapy in one system and enables the early detection of disease, simultaneous monitoring and targeted therapy with minimal toxicity, and provides a novel targeting therapeutic strategy for pancreatic cancer. In most clinical imaging modalities, magnetic resonance imaging (MRI) is a widely used tool in pharmaceutical research due to its excellent soft tissue contrast property that provides three-dimensional (3D) anatomic images with high spatial resolution. Colloidal iron oxide nanoparticles such as superparamagnetic iron oxide (SPIO) and ultrasmall superparamagnetic iron oxide (USPIO) have been extensively investigated as MRI contrast agents with low toxicity and more potent contrast properties, and as carriers for targeted drug delivery in theranostic medicine studies. ${ }^{8}$ However, their lack of specificity reduces accumulation in target tissues, thus limiting their current use as therapeutics and as diagnostic agents for principally liver, lymph nodes, and the gastrointestinal tract.

How to improve the specificity of iron oxide nanoparticles to target tissues and how to integrate the therapeutic agents with iron oxide nanoparticles are the most important research points. Direct use of iron oxide nanoparticle as a drug carrier and modification of the particle surface such as attachment of targeting ligands would reduce the superparamagnetic properties of iron oxide nanoparticles. ${ }^{9}$ Besides, receptors specifically binding to the targeting ligands are usually expressed in a very low concentration in vivo. An amplification strategy is required to improve the MRI sensibility. Nanoimmunoliposome has been proved to be a promising carrier with many advantages for targeted contrast agents. ${ }^{10-13}$ First, the in vivo behavior of liposomes has been well established with processes such as PEGylation resulting in long circulation time. ${ }^{14,15}$ Second, liposomes have the ability to encapsulate a large number of contrast agents and deliver them together to a target site in vivo, avoiding the dilution of the body fluid. Third, it is convenient to couple the targeting ligand to the surface of the liposome to achieve specific cellular recognition and this process does not interfere with the inherent superparamagnetic property of iron oxide nanoparticles. Fourth, a wide variety of therapeutic agents, including small molecules, ${ }^{16}$ proteins, ${ }^{17}$ peptides, ${ }^{18}$ DNA, ${ }^{19}$ and RNA, ${ }^{20}$ can be all encapsulated in liposomes. Although liposomes have many advantages as the carrier for targeted contrast agents, but there are still great challenges in how to obtain a stable liposomal solution with high loading of iron oxide nanoparticles. Based on the above knowledge, we constructed an anti-MSLN antibody-conjugated multifunctional nanoimmunoliposome drug-delivery system (referred to as M-PLDU) to encapsulate iron oxide nanoparticles and chemotherapy drug doxorubicin (DOX) for MRI imaging and pancreatic cancer-targeted therapy. To minimize the liposome size, USPIO (SHU555C, Superavist ${ }^{\circledR}$, Bayer Schering Pharma AG, Berlin, Germany) was selected as the MRI contrast agent, which is composed of monocrystalline magnetite $\left(\mathrm{Fe}_{3} \mathrm{O}_{4}\right)$ cores about $5 \mathrm{~nm}$ in diameter coated with an inert dextran layer giving it a hydrodynamic diameter of about $20 \mathrm{~nm}$. In order to increase the loading efficiency and stability of USPIO encapsulated in liposome, we applied the transient binding of the oxidized dextran with aminogroups of aminophosphalipids at $\mathrm{pH} 8.0$ and reverse-phase evaporation (REV) method to prepare the PEGlyated stealth liposome. To achieve the pancreatic cancer targeted effect, the prepared PEGlyated liposomes were decorated with anti-MSLN antibody by the post-insertion method. Both the therapeutic effect and the MRI capability of the novel multifunctional drug-delivery system were evaluated in Panc-1 cell line as well as in a pancreatic tumor xenograft athymic mouse model. The purpose of this study was to provide a multifunctional targeted drug-delivery system for both early detection of pancreatic cancer and MRI visible targeting and real-time monitoring of the therapeutic efficacy.

\section{Materials and methods Materials}

Materials used in this study included soybean phosphatidylcholine $\left(\mathrm{S}_{100} \mathrm{PC}\right)$ (Lipoid Co, Ludwigshafen, Germany); dioleoylphosphatidylethanolamine (DOPE), 1,2-distearoyl-sn-glycero-3-phosphoe- 
thanolamine-N-[methoxy(polyethyleneglycol)-2000] (mPEG 2000 -DSPE), 1,2-distearoyl-sn-glycero3-phosphoethanolamine-N-[maleimide (polyethylene glycol)-2000] (Mal-PEG ${ }_{2000}$-DSPE) and 1,2-dioleoyl-snglycero-3-phosphoethanolamine-N-carboxyfluorescein (CFPE) (Avanti Polar Lipids, Alabaster, AL); cholesterol (Chol), 3-(4, 5-dimethylthiazol-2-ly)-2,5-diphenyl-tetrazolium bromide (MTT), 2-iminothiolane (Traut's reagent) and Sephadex G-25 (Sigma-Aldrich, St Louis, MO); Dulbecco's modified Eagle's medium (DMEM), fetal bovine serum (FBS), penicillin, streptomycin and anti-MSLN mAb (antiMSLN) (Invitrogen, Carlsbad, CA); BCA protein assay reagent (Solarbio, Beijing, China); DOX hydrochloride (Melone Co, Dalian, China); and dextran-coated-USPIOs (SHU555C, Superavist $^{\circledR}$ ). All the other organic reagents were of analytical grade and purchased from Sinopharm (Shanghai, China).

\section{Cell lines}

Panc-1 human pancreatic cancer carcinoma cell line was available in the cell store of the Chinese Academy of Sciences and used for in vitro and in vivo experiments. This cell line can stably express a tumor differentiation antigen (cell surface glycoprotein) known as MSLN (data not shown). Panc-1 cells cultivated for all experiments were maintained in DMEM supplemented with $10 \% \mathrm{FBS}, 100 \mathrm{U} / \mathrm{mL}$ penicillin, $100 \mu \mathrm{g} / \mathrm{mL}$ streptomycin, and $4 \mathrm{mM} 1$-glutamine at $37^{\circ} \mathrm{C}$ in a humidified atmosphere containing $5 \% \mathrm{CO}_{2}$.

\section{Preparation of PLDU}

PEGylated liposomes composed of $\mathrm{S}_{100} \mathrm{PC}$ :DOPE:Chol: $\mathrm{mPEG}_{2000}$-DSPE (molar ratio, 6:1:2:1) were prepared by a transient binding and REV method as described previously with minor modifications..$^{21}$ First, the dextran on the surface of USPIOs was oxidized to generate aldehyde groups, which can attach to the aminogroups of aminophospholipids DOPE during liposome preparation in high $\mathrm{pH}$ condition, thus increasing the entrapment and stability of USPIOs in liposomes. Briefly, $2 \mathrm{~mL}$ USPIO solution at a concentration of $14 \mathrm{mg} \mathrm{Fe} / \mathrm{mL}$ was treated with $15 \mathrm{mg}$ sodium periodate (pH 6.0) for 30 minutes. After that, $20 \mathrm{mM}$ ethylene glycol was added into the solution to terminate the reaction. Then the USPIO solution was desalted using a PD10 column. After that, the total lipids (TL, ie, $\mathrm{S}_{100} \mathrm{PC}$, DOPE, mPEG2000DSPE, and Chol) were dissolved in chloroform and added to a rotary evaporator to form a lipid film layer under reduced pressure. $0.5 \mathrm{~mL}$ oxidized and purified USPIO solution was added into the round-bottom flask and vortexed. The obtained mixture was added with DOX solution (100 g drug/mol total lipids) and vortexed at $\mathrm{pH}$ 8.0. Then a threefold volume of chloroform and diethyl ether (1:2) was added to the vortex mixture and sonicated for about 10 minutes in an ice bath $\left(0^{\circ} \mathrm{C}\right)$ until a stable $\mathrm{W} / \mathrm{O}$ emulsion formed. This emulsion was then applied onto the rotary evaporator to remove the organic solvents under low pressure $(260-400 \mathrm{mmHg})$ at $37^{\circ} \mathrm{C}$. A coarse PEGylated liposome suspension was formed by gentle sonication in a Transonic Digitals bath sonifier (Elma, Germany) for 5 minutes. To remove USPIOs attached to the outer surface of the liposomes, the liposomal suspension was further dialyzed against $10 \mathrm{mM}$ phosphate-buffered saline (PBS) with $150 \mathrm{mM} \mathrm{NaCl}$ for 3 hours at $\mathrm{pH} 4.5$, which was the result of an instability of the Schiff bond at acidic $\mathrm{pH}$ value. Then, the nontrapped USPIOs and DOX were removed from the liposome suspension by centrifugation at $8000 \mathrm{~g}$ for 20 minutes and the liposome pellet was washed twice with PBS. After that, the pellet was resuspended in $10 \mathrm{mM}$ PBS and $150 \mathrm{mM} \mathrm{NaCl}(\mathrm{pH}$ 8.0). The liposomes were extruded 10-15 times per pore size through two pore-sized polycarbonate membranes (400 nm to $200 \mathrm{~nm}$ ) (Nucleopore; Whatman, Maidstone, UK) using a handheld extruder (Avestin, Ottawa, Canada) at room temperature. Finally, the purified and uniformed PEGylated liposomes were stored tightly at $4{ }^{\circ} \mathrm{C}$ for further experiments. For the fluorescence labeled liposome preparation, $0.4 \mathrm{~mol} \% \mathrm{CFPE}$ relative to the total lipid was incorporated into the lipid mixture.

\section{Conjugation of anti-MSLN monoclonal antibody}

Anti-MSLN mAb was conjugated to PLDU by post-insertion method as described previously. ${ }^{22,23}$ First, an anti-MSLN $\mathrm{mAb}$ was incubated with Traut's reagent at a molar ratio of 1:100 in PBS ( $\mathrm{pH}$ 7.4) for 2 hours for antibody thiolation. To remove excess Traut's reagent, the thiolated antibody was further dialyzed in PBS (pH 7.4) with 5 mM EDTA for 24 hours. Then the number of sulfhydryl groups in the thiolated antibody was determined using Ellmann's reagent. The concentration of the thiolated antibody was also measured by BCA Protein Assay Reagent (Pierce, Rockford, IL). To prepare anti-MSLN mAb-conjugated PEGylated liposomes, $50 \mathrm{~mol} \% \mathrm{mPEG}_{2000^{-}}$-DSPE was substituted by Mal- $\mathrm{PEG}_{2000^{-}}$ DSPE, so the composition of immunoliposomes was changed to $\mathrm{S}_{100}$ PC:DOPE:Chol:mPEG ${ }_{2000}$-DSPE:Mal-PEG ${ }_{2000}-\mathrm{DSPE}$ at a molar ratio of $6: 1: 2: 0.5: 0.5$. For post-insertion, $5 \mathrm{~mol} \%$ of Mal- $\mathrm{PEG}_{2000}$-DSPE relative to total lipids was dissolved in PBS ( $\mathrm{pH}$ 7.4), and then incubated with the thiolated antibody (molar ratio of Mal-PEG ${ }_{2000}$-DSPE to antibody, 40:1) 
overnight at room temperature with gentle shaking to form antibody-conjugated micelles. Finally, the micelles were inserted into preformed PEGylated liposomes by co-incubation at $37^{\circ} \mathrm{C}$ for 3 hours to obtain M-PLDUs.

\section{Particle size and zeta potential}

The prepared liposomes were diluted with deionized water, and then the mean hydrodynamic particle size and zeta potential were determined at $25^{\circ} \mathrm{C}$ using a Nano $\mathrm{S}$ zetasizer (Malvern Instruments, Malvern, UK). Each experiment was repeated three times.

\section{Transmission electron microscopy (TEM)}

The morphology of liposomes was observed by TEM using a JEM-2010 instrument (Jeol, Tokyo, Japan), working under an acceleration voltage of $200 \mathrm{kV}$. For the analysis, one drop of liposomal suspension diluted by deionized water was pipetted onto a carbon film supported by a copper grid. After deposition, the dispersion was blotted away with filter strips and then let dry in air at room temperature. The liposomes were observed soon after grid preparation.

\section{Assay of encapsulated iron oxide}

The amount of encapsulated iron oxide was determined based on ferrous ion by using O-phenanthroline method as described previously. ${ }^{24}$ Briefly, aliquots of $100 \mu \mathrm{L}$ of liposomal solutions (PLDU) before and after purification, as well as M-PLDU, were mixed with $100 \mu \mathrm{L} 5 \%$ Triton $\mathrm{X}-100$ to destruct the liposome structure, followed by addition of $500 \mu \mathrm{L}$ concentrated $\mathrm{HCl}$ to ionize the iron oxide; then $5 \mathrm{~mL} \mathrm{1 \%}$ hydroxylamine hydrochloride solution, $5 \mathrm{~mL}$ $0.1 \%$ O-phenanthroline solution, and $5 \mathrm{~mL}$ sodium acetate buffer ( $\mathrm{pH} 4.6$ ) were added. The mixture was diluted with deionized water to the final volume of $50 \mathrm{~mL}$. After standing for 15 minutes, the absorbance was read at the wavelength of $510 \mathrm{~nm}$ with a UV1102 spectrophotometer (Techcomp, Shanghai, China). The iron oxide encapsulation efficiency (EE) was calculated as the ration of the amount of iron in purified PEGlyated liposomes against the total amount of iron in coarse PEGlyated liposomes before purification.

\section{Evaluation of DOX EE}

The DOX encapsulated in liposomes was quantitated by UV spectrometry at its maximum absorbance wavelength of $480 \mathrm{~nm}$. Briefly, the coarse liposome suspension was centrifuged at $8000 \mathrm{~g}$ for 20 minutes and the liposome pellet was washed twice with PBS. Then the total supernatant of each centrifugation was pooled and quantitated as the mass of unencapsulated DOX. Meanwhile, an equal volume of coarse liposome suspensions was directly dissolved in Triton X-100 (5\%, V/V) to measure total DOX. The DOX encapsulation efficiency was calculated using the following formula $\left(\mathrm{DOX}_{\mathrm{T}}\right.$ and $\mathrm{DOX}_{\mathrm{U}}$ were denoted as the mass of total DOX and unencapsulated DOX, respectively)

$$
\text { Encapsulation efficiency } \%=\frac{\mathrm{DOX}_{\mathrm{T}}-\mathrm{DOX}_{\mathrm{U}}}{\mathrm{DOX}_{\mathrm{T}}} \times 100 \%
$$

\section{Evaluation of anti-MSLN mAb integrity}

The integrity of anti-MSLN antibody after thiolation and conjugation with PLDU was evaluated by sodium dodecyl sulfate-polyacrylamide gel electrophoresis (SDS-PAGE) on $12 \%$ Tris- $\mathrm{HCl}$ minigel.

\section{MRI properties of M-PLDUs in vitro}

M-PLDUs and USPIOs were diluted with PBS ( $\mathrm{pH} 7.4)$ to six different iron concentrations $(0,0.05,0.1,0.2,0.4$, and $0.8 \mathrm{mM}$ ) and MRI was performed using a clinical 3.0 $\mathrm{T}$ whole-body magnetic resonance system (Siemens Trio, Erlangen, Germany). Multislice $\mathrm{T}_{2}$-weighted images were acquired at various echo times (TE) from $12 \mathrm{~ms}$ to $144 \mathrm{~ms}$ with a repetition time (TR) of $2500 \mathrm{~ms}$ using the following scan parameters: field of view $(\mathrm{FOV})=150 \times 150 \mathrm{~mm}^{2}$, matrix size $=256 \times 256$, and slice thickness $=1.5 \mathrm{~mm}$. After image acquisition, the magnitudes of image signal intensities were inferred within manually drawn regions of interest (ROIs) by ImageJ software (National Institutes of Health, Bethesda, MD) for each sample. Relaxivity rates $\mathrm{R}_{2}\left(1 / \mathrm{T}_{2}\right)$ were calculated from signal intensities using the following formula (SI, signal intensity; $\mathrm{S}_{0}$, constant):

$$
\mathrm{SI}=\mathrm{S}_{\mathrm{o}} \cdot \exp \left(-\mathrm{R}_{2} \cdot \mathrm{T}_{\mathrm{E}}\right)
$$

Each value of $\mathrm{R}_{2}$ was calculated with different iron concentrations. $T_{2}$ relaxivity, $r_{2}$ was calculated as slope from a plot of R2 versus iron concentration to compare MRI sensitivity of M-PLDUs and USPIOs.

\section{Cell viability assay}

The MTT viability assay was used to evaluate the cytotoxicity of free DOX (FD), M-PLDUs and PLDUs against Panc-1 cells. Briefly, cells were seeded in 96-well plates at $1.0 \times 10^{4}$ cells/well and incubated for 24 hours at $37^{\circ} \mathrm{C}(5 \%$ $\mathrm{CO}_{2}$ and saturated humidity) with culture medium. After cell attachment and growth resumption, the medium was replaced with $200 \mu \mathrm{L}$ fresh culture medium (10\% FBS) containing 
FD, M-PLDUs, and PLDUs in increasing concentrations $(0.17-22.07 \mu \mathrm{M})$ of DOX and incubation was continued for another 24 hours. The control group was treated with the same volume of PBS (pH 7.4). Then, $20 \mu \mathrm{L}$ MTT solution $(5 \mathrm{mg} / \mathrm{mL})$ was added to each well. After incubation with cells for 4 hours, the unreduced MTT and culture medium were discarded, followed by addition of $150 \mu \mathrm{L}$ dimethylsulfoxide (DMSO) to dissolve the formazan crystals. Plates were then gently shaken for 10 minutes, and subsequent absorbance was read at $570 \mathrm{~nm}$ using an ELISA plate reader. To evaluate the possible cytotoxic effect of USPIOs, cells were further incubated with USPIOs at different iron concentrations $(10-160 \mu \mathrm{M})$ according to the above method. Each assay was repeated three times. The data were presented as the percentage of viable cells against that of untreated control cells.

\section{Cellular uptake study by confocal laser scanning microscopy}

Cellular internalization of fluorescence-labeled liposomes in Panc-1 cells was evaluated by confocal laser scanning microscopy using a Leica TCS SP5 AOBS confocal microscopy system (Leica, Wetzlar, Germany). Briefly, Panc- 1 cells were seeded at a density of $5 \times 10^{4}$ cells/ well on $35 \mathrm{~mm}$ dishes and cultured for 24 hours before the experiment. To study receptor-mediated M-PLDU uptake, cells were further incubated with fluorescence-labeled M-PLDUs or PLDUs (400 $\mathrm{nmol} \mathrm{TL} / \mathrm{mL})$ in serum-free culture medium for 4 hours. After incubation, the culture medium was discarded, and cells were then washed twice with cold PBS to remove surface-bound liposomes, fixed with $4 \%$ paraformaldehyde for 10 minutes, and washed with PBS twice again. All fluorescence images were obtained by confocal microscopy.

\section{Determination of liposomal formulations transported within Panc-I cells}

The transportation of liposomal formulations within Panc-1 cells was quantitated by flow cytometry. Briefly, Panc-1 cells were seeded in 48-well plates at a density of $1 \times 10^{5}$ cells/well and grown for 24 hours. Then, cells were treated with M-PLDUs and PLDUs at 200 or $400 \mu \mathrm{M}$ of total lipids for additional 24 hours at $37^{\circ} \mathrm{C}$. Cells were trypsinized, washed twice with PBS and then analyzed using a FACScan flow cytometer (Becton Dickinson, San Jose, CA). All fluorescence measurements were collected as a logarithmic signal. The mean fluorescence intensity (MFI) was expressed as fluorescence data of liposomal formulations transported within Panc-1 cells.

\section{Animal study}

Male athymic BALB/c nu/nu mice were purchased from Shanghai SLAC Laboratory Animal Co, Ltd (Shanghai, China). Mice were bred and housed under specific pathogenfree (SPF) conditions and provided with autoclaved water and mouse chow ad libitum. All animal procedures were performed in accordance with the guideline of the Animal Protection and Care Committee of the Second Military Medical University, Shanghai, People's Republic of China.

\section{In vivo $M R I$ study}

$\mathrm{BALB} / \mathrm{c}$ nu/nu mice (male, 4 6 weeks) weighing 18-22 g were inoculated subcutaneously on the back of the neck with $5 \times 10^{6}$ Panc- 1 cells in $0.2 \mathrm{~mL}$ serum-free DMEM medium. When the tumor volume reached the size about $400 \mathrm{~mm}^{3}$, the mice were subjected to imaging study. The 16 mice were equally randomized to four groups: one group receiving an intravenous injection of the saline solution via the tail vein as control, and the other three groups receiving intravenous injection of aqueous dispersion of USPIOs, PLDUs, and M-PLDUs, respectively at a dose of $100 \mu \mathrm{mol} \mathrm{Fe} / \mathrm{kg}$ via the tail vein. Before the MRI experiments, the mice were anesthetized with $0.2 \mathrm{~mL}$ $4 \%$ chloral hydrate intraperitoneally, and the tumor site was positioned in the center of the coil. Then the animals were imaged prior to injection and 4 hours postinjection with a 3 T MR scanner (Siemens Trio) using a surface coil with $\mathrm{T}_{2}$-weighted spin echo sequence $(\mathrm{TR}=2500 \mathrm{~ms}$, $\mathrm{TE}=30 \mathrm{~ms}, \mathrm{FOV}=40 \times 40 \mathrm{~mm}^{2}$, matrix $=512 \times 512$, slice thickness $=0.8 \mathrm{~mm}$ ). The image signal intensity of the tumors was also inferred by ImageJ.

\section{In vivo antitumor activity assay}

The tumor was induced in BALB/c nu/nu mice by injecting approximately $5 \times 10^{6}$ Panc- 1 tumor cells subcutaneously. When the tumor volume reached about $50-60 \mathrm{~mm}^{3}$, $16 \mathrm{BALB} / \mathrm{c}$ nu/nu mice bearing Panc-1 pancreatic cancer xenografts were randomized to four different experimental groups. The mice of each group were intravenously injected with FD, PLDUs, M-PLDUs, or an equivalent volume of saline solution via the tail vein at a total DOX dose of $6 \mathrm{mg} / \mathrm{kg}$ ( $2 \mathrm{mg} / \mathrm{kg}$, once a week). The tumor size was measured with a caliper and the mice were weighed thrice weekly. The tumor volume was calculated according to the formula: tumor volume $=0.52 \mathrm{~L} \cdot \mathrm{W}^{2}$, where $\mathrm{L}$ is 
the length and $\mathrm{W}$ is the width of the tumor. After 3 weeks, all mice were sacrificed by vertebral dislocation to harvest the tumors.

\section{Biodistribution study of DOX in tumor- bearing mice}

The tumor-bearing BALB/c nu/nu mice with a tumor volume about $400 \mathrm{~mm}^{3}$ were injected with aqueous dispersion of FD, PLDUs, and M-PLDUs at a single DOX dose of $2 \mathrm{mg} / \mathrm{kg}$ via the tail vein. After 24 hours, the mice were sacrificed and the heart, kidney, spleen, lung, liver, and tumors were excised immediately, lightly blotted to remove any excess blood, and weighed to measure the amount of DOX. The tissue specimens were homogenized and extracted with chloroform/ methanol (4:1, v/v), and the DOX extractions were subjected to high-pressure liquid chromatography (HPLC) analysis.

The HPLC system consisted of an Agilent 1100 pump, an Agilent $1200 \mathrm{FLD}$ fluorescence detector $\left(\lambda_{\mathrm{Ex} / \mathrm{Em}}=470 / 550 \mathrm{~nm}\right.$; Agilent, Palo Alto, CA), and a Dikma Diamonsil ${ }^{\circledR} \mathrm{C} 18$ column $(5 \mu, 250 \times 4.6 \mathrm{~mm}$; Dikma, Shaghai, China). The mobile phase was methanol:water:acetic acid $(50: 45: 5, \mathrm{v} / \mathrm{v}){ }^{25}$ The flow rate was $1.0 \mathrm{~mL} / \mathrm{minute}$ and the column temperature was $30^{\circ} \mathrm{C}$. Measurements were made using the ratio of the peak area of DOX to that of an internal standard daunomycin.

\section{Statistical analysis}

All values are represented as mean \pm standard deviation. Direct comparison between two groups was conducted by Student's unpaired $t$-test. A $P$ value below 0.05 was considered significant for all analyses.

\section{Results and discussion Characterization of M-PLDUs}

A stable and nonaggregated liposomal solution with high payload of contrast agents is prerequisite for improving MRI sensitivity of tumor tissue in vivo. USPIOs are colloidal particles and it proved difficult to achieve this goal with a common liposome preparation method, such as the film dispersion method (the EE was less than $15 \%$ ). ${ }^{26}$ In this study, through transient binding and REV method we successfully obtained stable and nonaggregated PEGlyated liposomes with simultaneously loading of USPIOs and DOX, and by a postinsertion method, the anti-MSLN antibody was successfully conjugated to the terminus of PEG chain on the liposome surface. The scheme for constructing anti-MSLN-conjugated PEGylated liposomes was presented in Figure 1. The prepared M-PLDU colloidal liposomal solution was stable without evidence of aggregation and as displayed in Figure 2 (top), the dynamic laser light scattering (DLS) revealed a mean

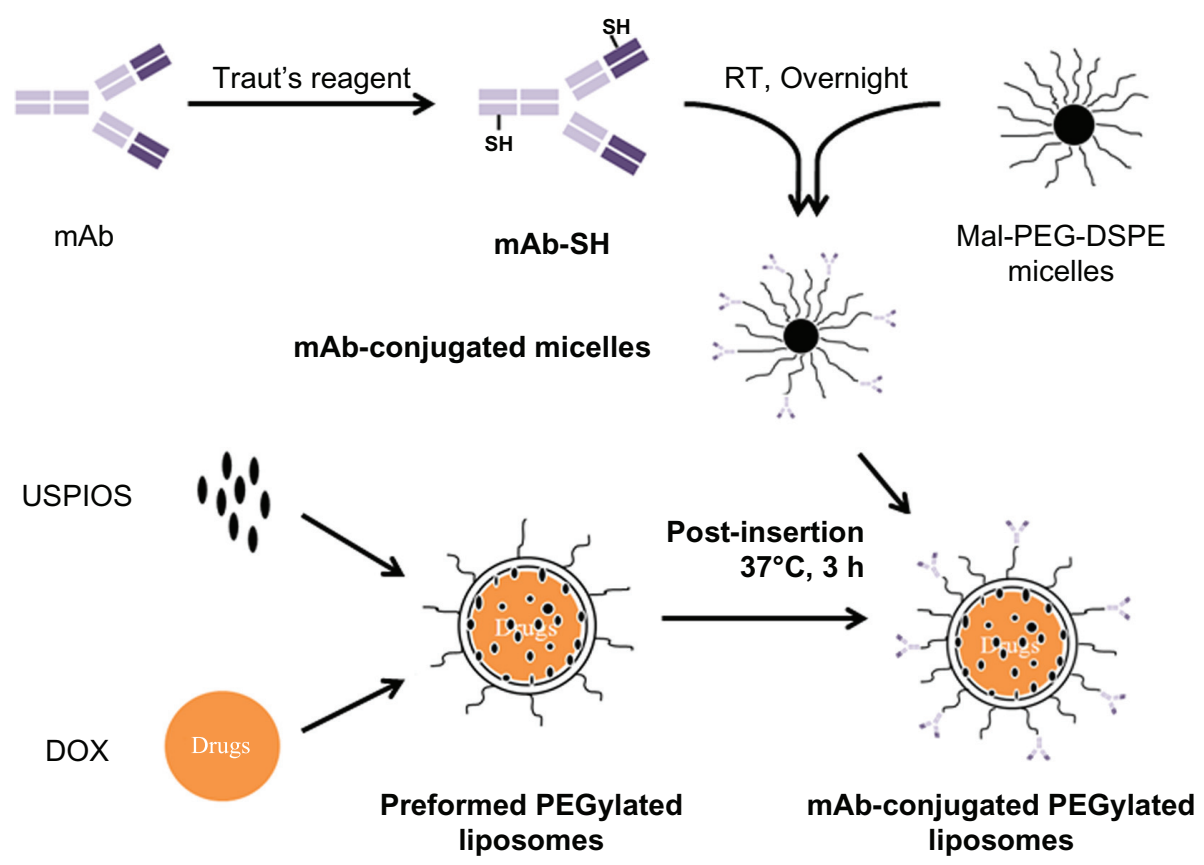

Figure I Schemes for constructing anti-MSLN-conjugated PEGylated liposomes.

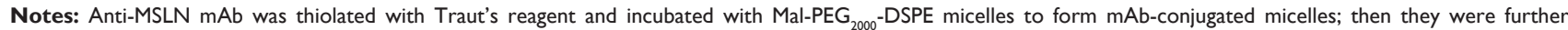
incubated with the preformed PEGylated liposomes at $37^{\circ} \mathrm{C}$ for 3 hours to obtain anti-MSLN-conjugated PEGylated liposomes. The PEGylated immunoliposomes retain the integrity of anti-MSLN mAb and are available for targeting.

Abbreviations: MSLN, mesothelin; PEG, polyethyleneglycol; Mal-PEG2000-DSPE, I.2 distearoyl-sn-glcero-3-phosphoethanolamine-N-[malemide(polyethyleneglycol)-2000]; DOX, doxorubicin; mAb, monoclonal antibody; USPIOs, ultrasmall superparamagnetic iron oxides. 

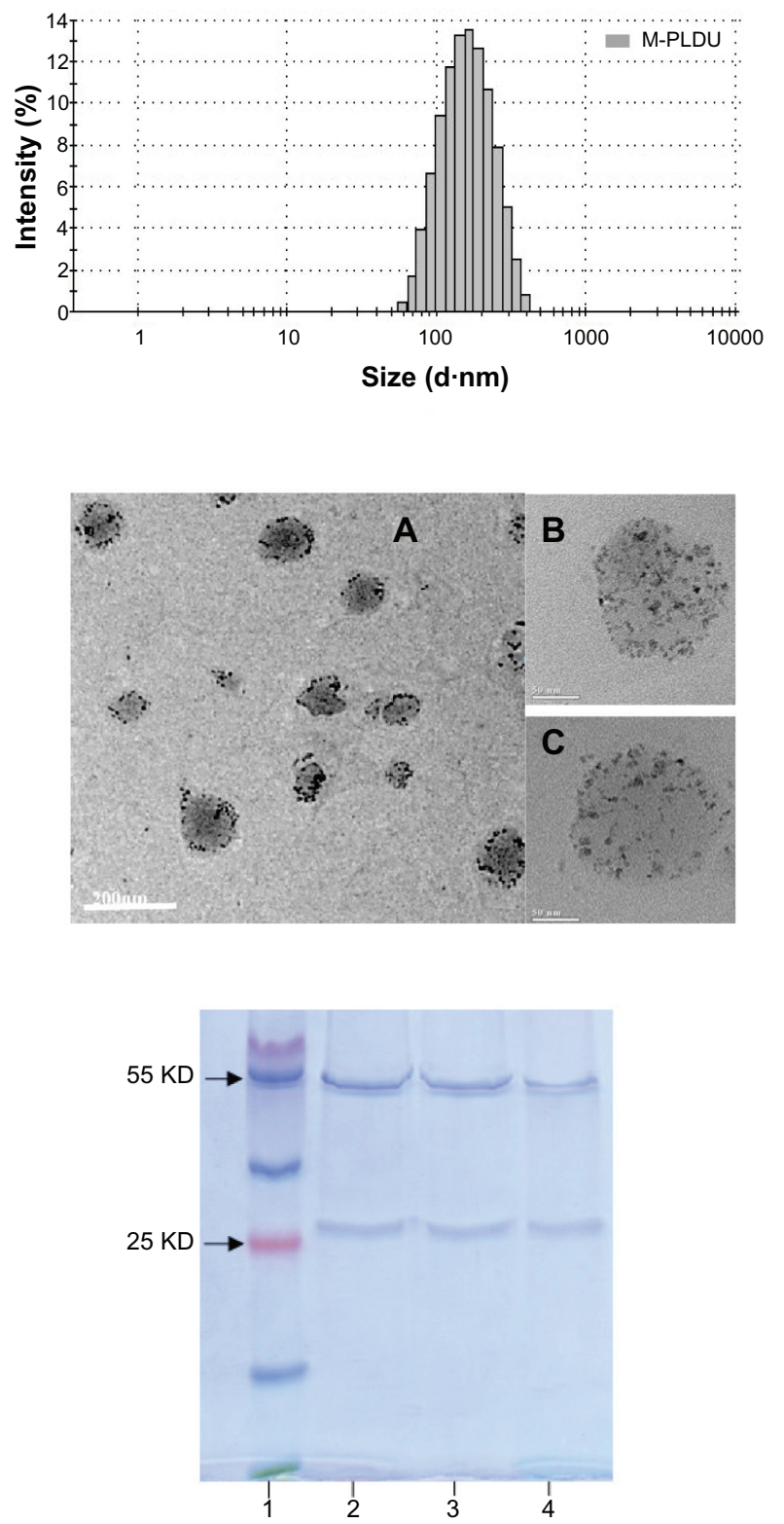

Figure 2 Characteristics of M-PLDU. Top: Hydrodynamic particle size distribution of M-PLDUs in water measured by dynamic laser light scattering (DLS). Middle: Transmission electron micrographs (TEM) of M-PLDUs after purification. The dark dots in the images represent the cores of USPIOs. Total magnification: $\times 200000$, Bar $=200 \mathrm{~nm}(\mathbf{A}) ; \times 820000, \mathrm{Bar}=50 \mathrm{~nm}$ (B and C). Bottom: SDS-PAGE analysis of anti-MSLN mAb. Lane I: protein marker; lane 2: anti-MSLN mAb; lane 3: thiolated anti-MSLN mAb after reduction using Traut's reagent and purification using dialysis; lane 4: anti-MSLN mAb after incorporation into PEGylated liposomes.

Abbreviations: MSLN, mesothelin; PEG, polyethyleneglycol; mAb, monoclonal antibody; SDS-PAGE, sodium dodecyl sulfate-polyacrylamide electrophoresis; USPIOs, ultrasmall superparamagnetic iron oxides.

hydrodynamic size of $175 \sim 185 \mathrm{~nm}$ with a polydispersity index of about 0.203 . The hydrodynamic size of the PLDU without antibody conjugation was about 160 170 nm with a polydispersity index of 0.182 , which confirms that anti-MSLN conjugation did not significantly increase the liposome size. The zeta potential of the M-PLDUs measured using Nano
S zetasizer (Malvern Instruments) was about $-28 \sim-30 \mathrm{mV}$. TEM results confirmed that all liposomes were spherical in shape, and few free USPIOs or aggregates were present in the purified M-PLDU dispersions (Figure 2, middle, A). TEM showed there are many USPIO particles in one liposome and those USPIO particles were either successfully bound to the interior layer of lipid bilayer of liposomes by covalent attachment or passively encapsulated in the internal aqueous phase (Figure 2, middle, B, C). According to previous studies, after intravenous injection, the PEGlyated particles with a diameter below $200 \mathrm{~nm}$ can move freely in the blood circulation, while particles larger than 200-250 nm often become opsonized and are removed from blood circulation quickly. ${ }^{27,28}$ The prepared M-PLDUs were uniform with a particle size less than $200 \mathrm{~nm}$, which assured enough circulation time for them to accumulate in the tumor tissue. Meanwhile, it has been found that the cut-off size of the leaky vasculature in most tumors is $200-600 \mathrm{~nm},{ }^{29}$ which means that M-PLDU below $200 \mathrm{~nm}$ can easily accumulate in the tumor tissue by the enhanced permeability and retention (EPR) effect.

The concentration of iron in M-PLDU was calculated as $0.47 \pm 0.05 \mathrm{mg} / \mathrm{mL}$, and the EE of iron oxide in M-PLDU was up to $40.8 \% \pm 4.6 \%$. In our preliminary study, the EE of iron in liposomes prepared by film dispersion method was less than $15 \%$. So the transient binding method could increase the EE\% of iron in liposomes. The concentration of DOX in M-PLDU was $0.228 \pm 0.019 \mathrm{mg} / \mathrm{mL}$, and the EE of DOX in M-PLDU was $68.5 \% \pm 5.8 \%$. Because the $\mathrm{pH}$ value of internal aqueous phase in prepared liposomes was 8 , which was predetermined for formation of Schiff bond between oxidized USPIOs and DOPE, it's hard to achieve the active loading of DOX by the $\mathrm{pH}$ gradient method..$^{30} \mathrm{DOX}$ hydrochloride was encapsulated in liposomes simultaneously with USPIO solution. Meanwhile, the aldehyde groups on the surface of oxidized USPIOs were also able to attach to the aminogroups of DOX to increase the DOX encapsulation. After antibody thiolation and immunoliposome preparation, SDS-PAGE was used to evaluate the integrity of anti-MSLN mAb. The two distinct bands at $50 \mathrm{kDa}$ and $30 \mathrm{kDa}$ demonstrated the integral heavy chain and light chain (Figure 2, bottom), respectively, and confirmed that anti-MSLN mAb remained integral after incorporating into the PEGylated liposome.

\section{MRI of M-PLDUs in vitro}

To determine whether the oxidation procedure and liposome preparation process would eradicate the superparamagnetic properties of USPIOs, the MRIcapability and relaxation time of USPIOs with M-PLDU were compared in vitro by a 3.0 
A

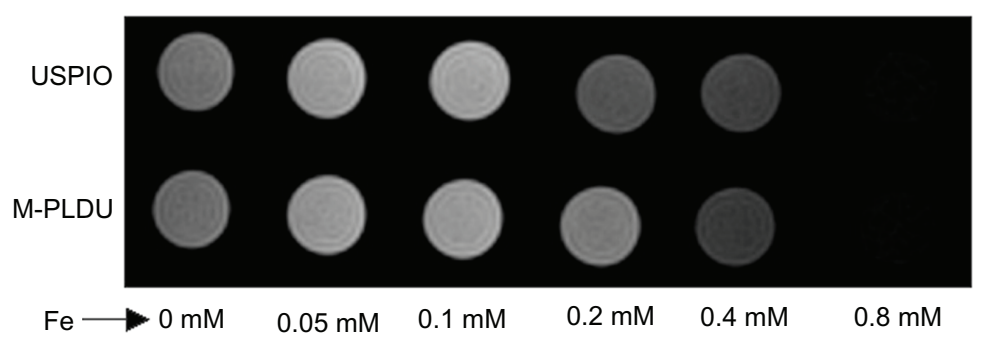

B
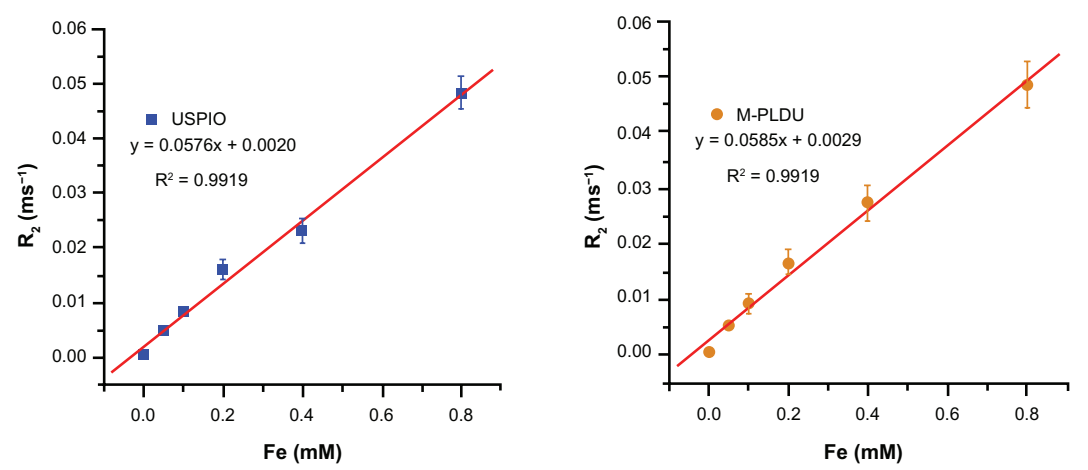

Figure 3 MRI properties of USPIOs and M-PLDUs in vitro: (A) signal intensity $\mathrm{T}_{2}$-weighted images (TR $=2500 \mathrm{~ms}$; TE $=36 \mathrm{~ms}$ ) of USPIOs and M-PLDUs in PBS at various iron concentrations at $25^{\circ} \mathrm{C}$; blank PBS was taken as a control; (B) $T_{2}$ relaxation rate $\left(R_{2}\right)$ of USPIOs and M-PLDUs vs iron concentration.

Note: Data as values obtained from curve fitting.

Abbreviations: MRI, magnetic resonance imaging; USPIOs, ultrasmall superparamagnetic iron oxides.

T magnetic resonance system. As shown in Figure 3A, with the iron concentration increasing in the M-PLDU group, the signal intensity gradually decreased compared with the blank wells. Meanwhile, the transverse relaxation time $\mathrm{T}_{2}$ was also gradually reduced from $188.7 \mathrm{~ms}$ to $21.3 \mathrm{~ms}$. The relaxation rate, $R_{2}=1 / T_{2}$, was linearly proportional to the iron concentration (Figure $3 B$ ). The slopes from a plot of $R_{2}$ versus iron concentration represent the $T_{2}$ relaxivities $\left(r_{2}\right)$ of M-PLDUs and USPIOs, and showed almost similar values (0.0585 $\mathrm{mM}^{-1} \mathrm{~ms}^{-1}$ and $0.0576 \mathrm{mM}^{-1} \mathrm{~ms}^{-1}$, respectively). These results demonstrated that the entrapment of USPIOs in liposomes and decoration of the liposome surface with anti-MSLN did not interfere with the inherent superparamagnetic properties of USPIOs. In fact, some studies even demonstrated that liposome entrapment could enhance the relaxivity of the iron oxide-based contrast agents. ${ }^{31,32}$ Liposomes may prove to be a promising nanocarrier for targeted delivery of $\mathrm{T}_{2}$ contrast agents.

\section{Cell viability assay}

Using the Panc-1 cell line as an in vitro cell model, the antitumor activity of M-PLDU was compared with PLDU and FD by MTT viability assay. In addition, the cytotoxicity of USPIOs on Panc-1 cell was also evaluated. As shown in the dose-response curves in Figure 4A, targeted M-PLDUs were more cytotoxic than nontargeted PLDUs. However, both liposomal preparations exhibited much less cytotoxicity as compared with FD. The half-maximal inhibitory concentration values were $0.53,1.95$, and $3.5 \mu \mathrm{M}$ for FD, M-PLDUs, and PLDUs, respectively, indicating that the antitumor activity of M-PLDU was 1.8 times that of PLDU. In addition, USPIOs showed no significant cytotoxicity on Panc-1 cells (Figure 4B). The viability of USPIO-treated cells was the same as that of PBS-treated cells. These results suggest that the antibody-mediated endocytosis contributed to the increased cytotoxicity of M-PLDU compared with nontargeted liposomes. However, both liposomal formulations showed less antitumor effect than FD solution. This may be because gradual release of DOX from M-PLDU or PLDU could not quickly reach the DNA target in the nucleus and kill cells at the same time during incubation.

\section{Fluorescence imaging by confocal laser scanning microscopy}

Cellular internalization and localization of CFPE-labeled liposomes were further studied using confocal laser scanning 

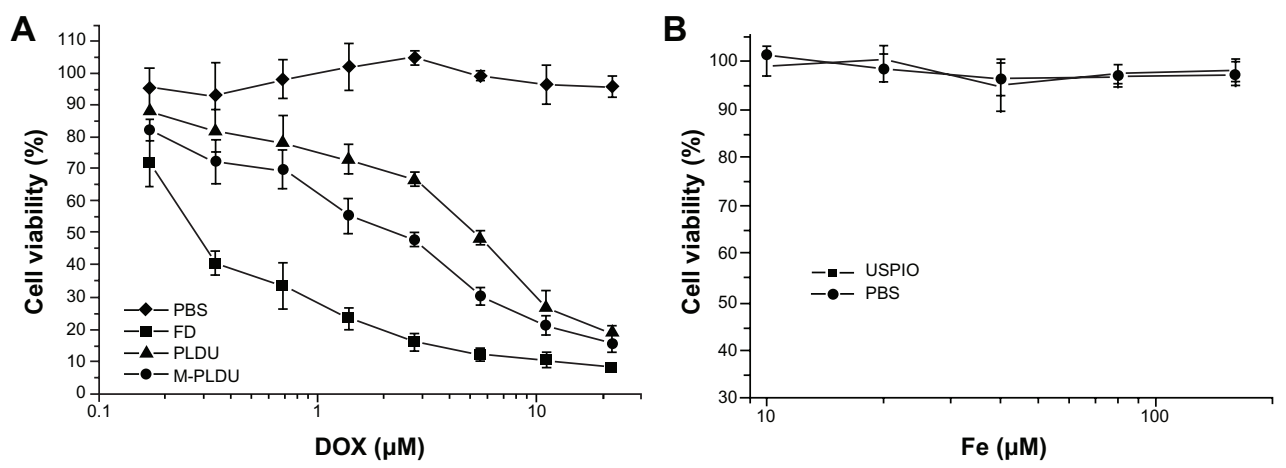

Figure 4 Dose response curves for various formulations against Panc-I cells. For in vitro cytotoxicity assay, Panc-I cells were treated with M-PLDUs, PLDUs, and FD at various DOX concentrations (A), or USPIOs at various Fe concentrations (B); PBS was set as control group in both experiments.

Notes: Cell viability was analyzed by MTT assay and calculated as a percentage of living cells. Each point represents the mean \pm SD $(n=3)$.

Abbreviations: FD, free doxorubicin; DOX, doxorubicin.

microscopy. Panc-1 cells were incubated with M-PLDUs or PLDUs at $37^{\circ} \mathrm{C}$ for 4 hours. Before fluorescence imaging, cells were washed twice to avoid nonspecific adhesion. Then, significant green fluorescence signals were observed in Panc-1 cells after being treated with M-PLDUs for 4 hours (Figure 5). However, the incubation of PLDUs with Panc-1 cells only showed very weak fluorescence signals. The results demonstrated that the conjugation of anti-MSLN could well improve the internalization of liposomes into Panc-1 cells.

\section{Determination of liposomal formulations transported within Panc-I cells}

The quantification of liposome formulations transported within Panc-1 cells was performed by flow cytometry.
Knowing that the degree of liposomes transported within cells depends on the liposomal specificity and concentration. Panc-1 cells in this study were set to incubate with two liposomal concentrations ( 200 and $400 \mu \mathrm{MTL}$ ) and formulations (M-PLDUs and PLDUs). Figure 6 shows that with the added liposomal concentration increasing at a constant rate, the number of liposomes transported within cells also increased. In addition, the amount of M-PLDUs transported within cells at the same liposomal concentration was higher than that of PLDUs $(P<0.05$ and 1.2- and 1.4-fold higher in 200 and $400 \mu \mathrm{M}$ TL, respectively). These results further showed that the anti-MSLN modification of liposomes played a very important role in increasing the transportation of liposomes within cells.

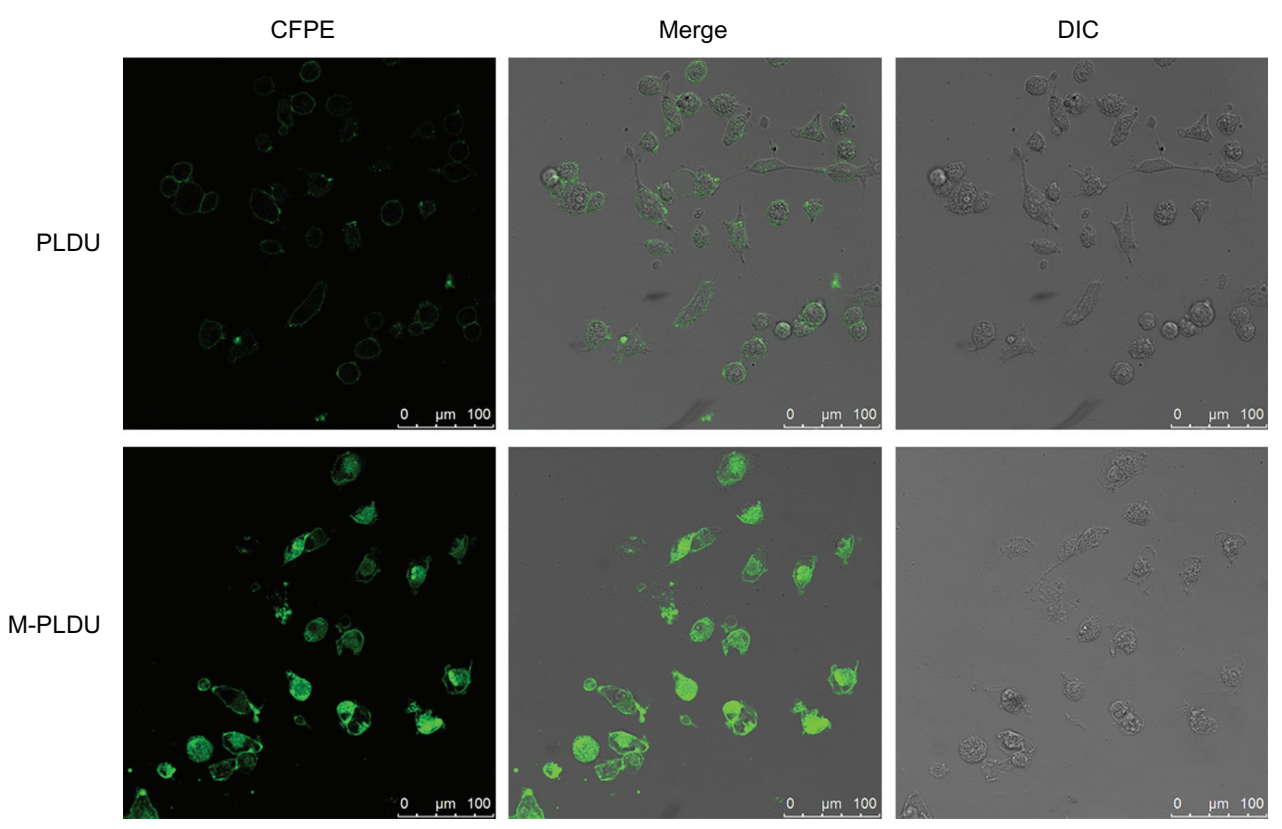

Figure 5 Cellular internalization images of fluorescently labeled liposomes by confocal laser scanning microscopy

Notes: Human pancreatic cancer Panc-I cells were incubated with PLDUs or M-PLDUs at $37^{\circ} \mathrm{C}$ for 4 hours, and then the same samples were fixed with $4 \%$ paraformaldehyde and observed under a confocal microscope to detect fluorescence. $(B a r=100 \mu \mathrm{m})$. 


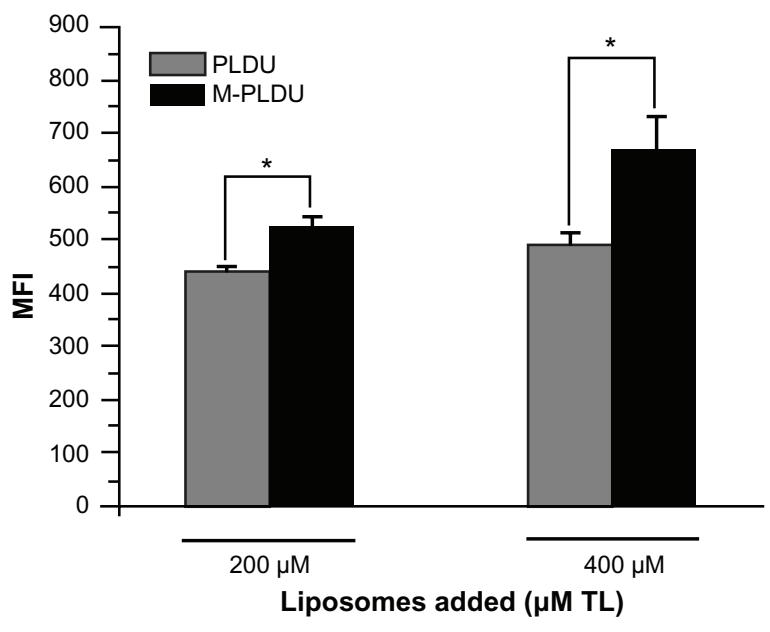

Figure 6 Quantification of liposomal formulations transported within Panc-I cells. Notes: Panc-I cells were seeded in 48-well plates at a density of $I \times 10^{5}$ cells per well for 24 hours. Then M-PLDUs or PLDUs labeled with CFPE were incubated with the cells for another 24 hours at $37^{\circ} \mathrm{C}$ (final total lipid concentration was $200 \mu \mathrm{M}$ or $400 \mu \mathrm{M})$. The cells were trypsinized and washed twice with PBS, and finally MFI was analyzed by FCM. Each point represents the mean $\pm S D(n=3$, $* P<0.05)$. Abbreviations: FCM, flow cytometer; MFI, mean fluorescence intensity.

\section{Targeting $M R I$ in vivo}

The signal intensity of a voxel of tissue is determined according to imaging parameters and inherent tissue properties, including hydrogen spin density, relaxation time and magnetic susceptibility. The magnetic resonance $\mathrm{T}_{2}$ contrast agents work predominantly by shortening transverse relaxation time $\mathrm{T}_{2}$, with the decrease of signal intensity on $\mathrm{T}_{2}$-weighted imaging. The process of $\mathrm{T}_{2}$ shortening is a remote effect that can enhance $\mathrm{T}_{2}$ relaxation with local magnetic field heterogeneity produced by $\mathrm{T}_{2}$-contrast agents. The degrees of relaxation enhancement can be interpreted as being related to the concentration of contrast agents in tissue. Figure 7A presents a subset of MRI images obtained from four different groups of animals before and 4 hours after treatment. The MRI images of xenografted tumors showed different degrees of SI drop in different treatment animal groups except that of the saline treatment group. For the USPIO group, only little SI change was observed after particle injection, while significant SI change was observed in M-PLDU and PLDU groups and M-PLDU group exhibited lower SI than did PLDU group. The lower SI value demonstrated the higher iron content within the tumor tissue. Then, the quantitative information on different liposomal targeting distributions in tumors was inferred from the ROIs of tumors by ImageJ, and the SI change $(\Delta \mathrm{SI}=$ SI before administration - SI after administration) was calculated. As shown in Figure 7B, the decrease in tumor SI induced by M-PLDUs $(\Delta \mathrm{SI}=145.98 \pm 20.45)$ or PLDUs $(\Delta \mathrm{SI}=75.69 \pm 14.53)$ was much more significant than that by USPIOs $(\Delta \mathrm{SI}=42.78 \pm 22.12)$ at $P<0.05$ or $P<0.01$, respectively. These results confirmed that the nanoimmunoliposome encapsulation of USPIOs can significantly improve the kinetic profile of USPIOs accumulation within the tumor tissues.

\section{Anticancer effect of M-PLDUs in vivo}

To evaluate the anticancer effect of M-PLDU in the animal model, BALB/c nu/nu mice bearing Panc-1 xenografts $\left(\sim 50-60 \mathrm{~mm}^{3}\right)$ were injected intravenously with FD or various liposomal formulations at a total DOX dosage of $6 \mathrm{mg} / \mathrm{kg}$ ( $2 \mathrm{mg} / \mathrm{kg}$ once a week). Mice were given saline solution as a control. Tumor growth inhibition curves in terms of mean tumor volume $\left(\mathrm{mm}^{3}\right)$ are presented in Figure 8A. The figure showed that the tumors grew almost linearly along with the culture time in all cases. In addition, tumor growth
A

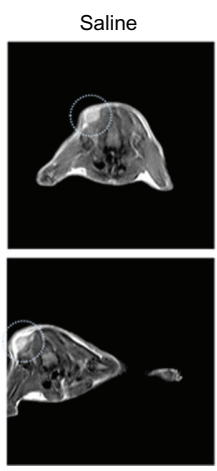

USPIO
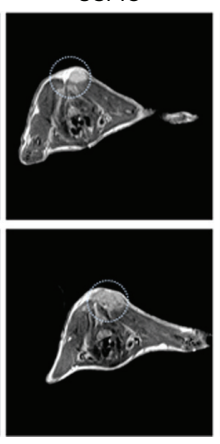

PLDU

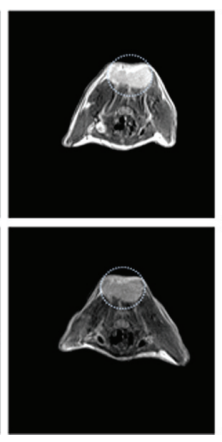

M-PLDU

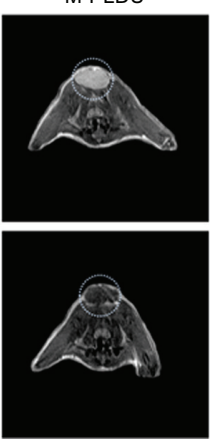

B

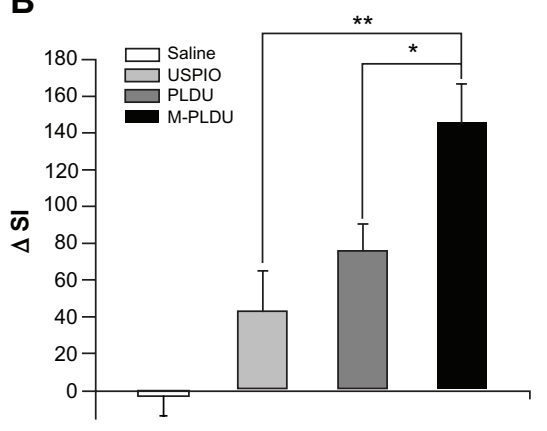

Figure $7 \mathrm{MRI}$ of tumors in vivo. (A) Representative subsets of $\mathrm{T}_{2}$-weighted MRI and (B) changes in signal intensity before and after injection of saline solution, USPIOs, PLDUs, and M-PLDUs.

Notes: MRI of the tumors was performed with a $3 T$ MR scanner (Siemens Trio) using a surface coil with $T_{2}$-weighted spin echo sequence $(T R=2500$ ms; $T E=30$ ms; FOV $=40 \times 40 \mathrm{~mm}^{2}$; matrix $=512 \times 512$; slice thickness $=0.8 \mathrm{~mm}$ ). Quantitative information in signal intensities was inferred by Image software $(\mathrm{n}=4$ in each group; $* P<0.05$, **P $<0.01)$.

Abbreviation: USPIOs, ultrasmall superparamagnetic iron oxides. 
A

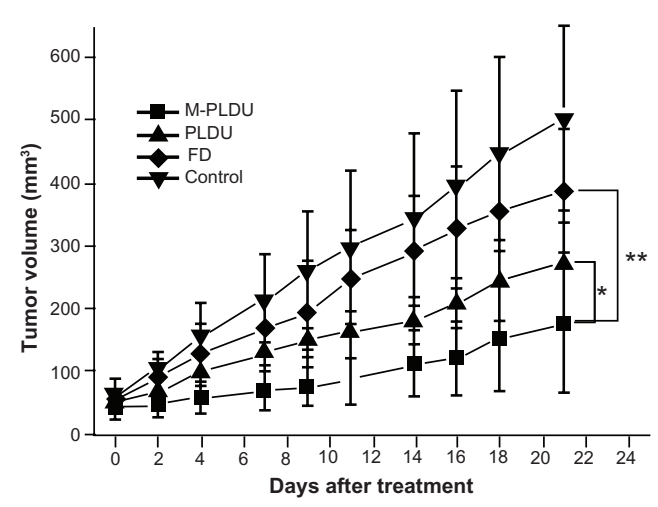

C

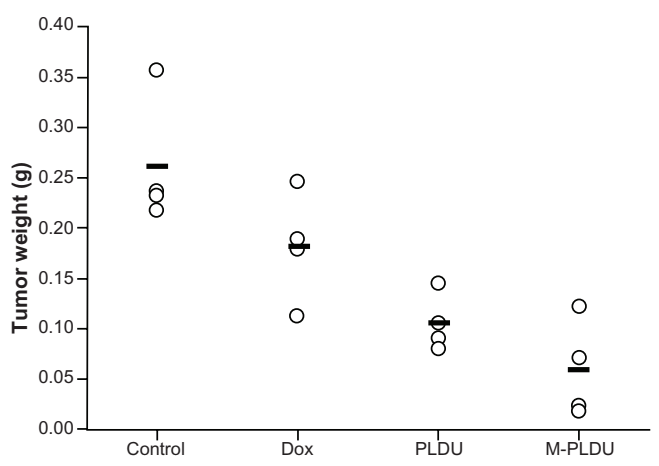

B

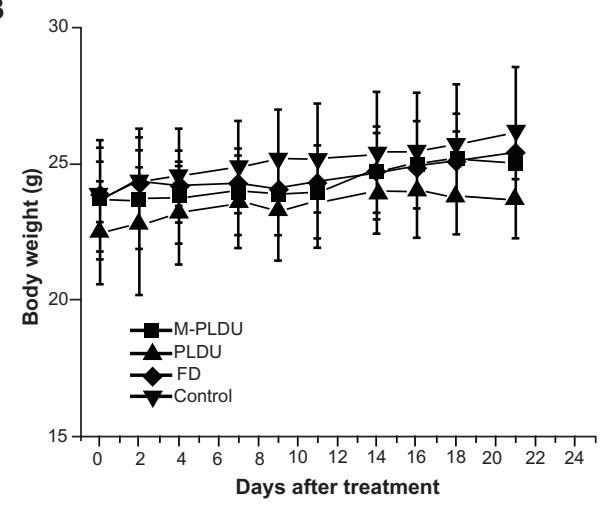

D

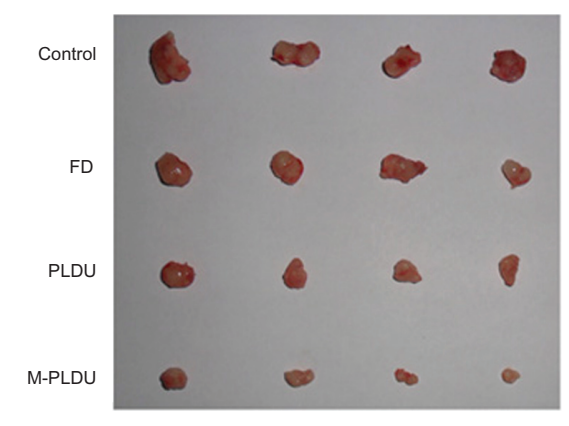

Figure 8 Therapeutic efficacy of M-PLDUs in human pancreatic cancer xenografts. (A) The tumor volume of mice after different treatment $(n=4$ in each group, $* P<0.05$, **P $<0.01$ ). (B) Mean body weight of each group. (C) Tumor weight at the end of the treatment. (D) Images of tumors collected in each group.

was effectively prevented by all the DOX formulations. The tumors in M-PLDU-treated mice were smaller than those in mice administered with PLDUs $(P<0.05)$ and FD $(P<0.01)$. The mean body weights are shown in Figure 8B. No significant body weight loss was observed in all the treated mice compared with the animals in the control group. By the end of the treatment, the final mean tumor weight in the mice treated with M-PLDUs was $0.06 \mathrm{~g}$ versus $0.10 \mathrm{~g}$ and $0.18 \mathrm{~g}$ in the mice treated with PLDUs and FD, respectively (Figure 8C and D). The results demonstrated that M-PLDU possessed the antitumor effect in vivo and the effect was improved compared with FD and PLDUs.

\section{Biodistribution of DOX in vivo}

To further confirm the therapeutic efficacy enhanced by M-PLDUs, drug accumulation in the tumor tissue was evaluated after 24 hours by injections with FD, PLDUs or M-PLDUs. The mean intratumor DOX concentration in M-PLDU group was 1.49-fold higher than that in the PLDU group, and 3.05-fold higher than that in the FD group, respectively (Figure 9). Meanwhile, the DOX concentration in the M-PLDU and PLDU groups was significantly higher than that of FD in the kidney, spleen, lung, and liver, while no significant increase in DOX concentration was observed in the heart of the M-PLDU and PLDU groups. These results confirmed that M-PLDU improves the tumor-targeting distribution of DOX and decreases the distribution of DOX in the heart, therefore improving the antitumor effect of DOX and reducing the cardiac toxicity of DOX.

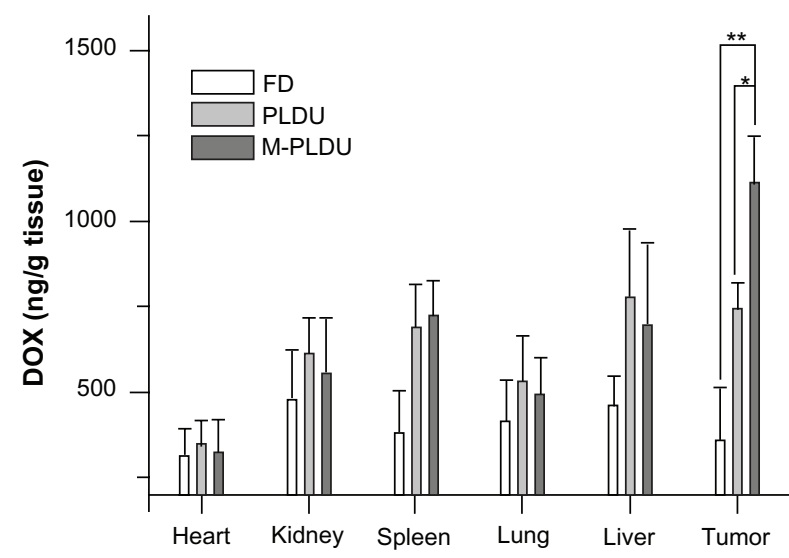

Figure 9 The biodistribution of different formulations in tumors and normal tissues. Notes: At 24 hours after injection, the DOX concentration was measured by an Agilent 1200 FLD fluorescence detector. The DOX concentration inside tumor tissue in M-PLDU group was higher than that in FD and PLDU groups $(n=4$ in each group; $* P<0.05, * * P<0.01$ )

Abbreviations: DOX, doxorubicin; FD, free doxorubicin. 


\section{Conclusion}

In this study, DOX and USPIOs were successfully coloaded into the liposomes with relatively high EE, and the antiMSLN mAbs were also well conjugated to the preformed PEGylated liposomes to develop immunoliposomes by the post-insertion method. This kind of targeting immunoliposomes against MSLN antigen should be an effective approach to deliver anticancer drugs and contrast agents to pancreatic cancer cells. Our results demonstrated that M-PLDUs were capable of specifically recognizing the MSLN-expressing cell surface, thus mediating endocytosis of liposomes in MSLN-expressing cells. In addition, M-PLDUs could well improve the therapeutic efficacy of DOX chemotherapy in vivo, and M-PLDUs were also proved to have excellent MRI properties and targeting ability in vivo. In conclusion, this MSLN-mediated immunoliposomal formulation has a promising potential for targeted anticancer drug therapy and for early MRI diagnosis of pancreatic cancer.

\section{Acknowledgments}

This work was financially supported by National Natural Science Foundation of China (81101141) and the Science and Technology Foundation of Shanghai Municipality of China (grant no 1052 nm03300). We thank Xiaoxia Du PhD in Shanghai Key Laboratory of Magnetic Resonance for her technical assistance.

\section{Disclosure}

The authors report no conflicts of interest in this work.

\section{References}

1. Jemal A, Bray F, Center MM, Ferlay J, Ward E, Forman D. Global cancer statistics. CA Cancer J Clin. 2011;61(2):69-90.

2. Pliarchopoulou K, Pectasides D. Pancreatic cancer: current and future treatment strategies. Cancer Treat Rev. 2009;35:431-436.

3. Argani P, Iacobuzio-Donahue C, Ryu B, et al. Mesothelin is overexpressed in the vast majority of ductal adenocarcinomas of the pancreas: identification of a new pancreatic cancer marker by serial analysis of gene expression (SAGE). Clin Cancer Res. 2001;7:3862-3862.

4. Ordóñez NG. Application of mesothelin immunostaining in tumor diagnosis. Am J Surg Pathol. 2003;27:1418-1428.

5. Roe OD, Creaney J, Lundgren S, et al. Mesothelin-related predictive and prognostic factors in malignant mesothelioma: a nested case-control study. Lung Cancer. 2008;61:235-243.

6. Hassan R, Bera T, Pastan I. Mesothelin: a new target for immunotherapy. Clin Cancer Res. 2004;10(12 Pt 1):3937-3942.

7. Sumer B, Gao J. Theranostic nanomedicine for cancer. Nanomedicine (Lond). 2008;3(2):137-140.

8. Sun C, Lee JS, Zhang M. Magnetic nanoparticles in MR imaging and drug delivery. Adv Drug Deliv Rev. 2008;60:1252-1265.

9. Di Marco M, Sadun C, Port M, Guilbert I, Couvreur P, Dubernet C. Physicochemical characterization of ultrasmall superparamagnetic iron oxide particles (USPIO) for biomedical application as MRI contrast agents. Int J Nanomedicine. 2007;2:609-622.
10. Yang C, Rait A, Pirollo KF, Dagata JA, Farkas N, Chang EH. Nanoimmunoliposome delivery of superparamagnetic iron oxide markedly enhances targeting and uptake in human cancer cells in vitro and in vivo. Nanomedicine. 2008;4:318-329.

11. Kozlowska D, Foran P, MacMahon P, Shelly MJ, Eustace S, O'Kennedy R. Molecular and magnetic resonance imaging: The value of immunoliposomes. Adv Drug Deliv Rev. 2009;61:1402-1411.

12. Torchilin VP. Recent advances with liposomes as pharmaceutical carriers. Nat Rev Drug Discov. 2005;4:145-160.

13. Frascione D, Diwoky C, Almer G, et al. Ultrasmall superparamagnetic iron oxide (USPIO)-based liposomes as magnetic resonance imaging probes. Int J Nanomedicine. 2012;7:2349-2359.

14. Maruyama K. Intracellular targeting delivery of liposomal drugs to solid tumors based on EPR effects. Adv Drug Deliv Rev. 2011;63: 161-169.

15. Yoshizawa Y, Kono Y, Ogawara K, Kimura T, Higaki K. PEG liposomalization of paclitaxel improved its in vivo disposition and anti-tumor efficacy. Int J Pharm. 2011;412(1-2):132-141.

16. Dass CR, Choong PF. Targeting of small molecule anticancer drugs to the tumour and its vasculature using cationic liposomes: lessons from gene therapy. Cancer Cell Int. 2006;6:17.

17. Meldrum KK, Burnett AL, Meng X, et al. Liposomal delivery of heat shock protein 72 into renal tubular cells blocks nuclear factor-kappaB activation, tumor necrosis factor-alpha production, and subsequent ischemia-induced apoptosis. Circ Res. 2003;92: 293-299.

18. Torchilin VP, Rammohan R, Weissig V, Levchenko TS. TAT peptide on the surface of liposomes affords their efficient intracellular delivery even at low temperature and in the presence of metabolic inhibitors. Proc Natl Acad Sci U S A. 2001;98:8786-8791.

19. Reimer DL, Kong S, Monck M, et al. Liposomal lipid and plasmid DNA delivery to B16/BL6 tumors after intraperitoneal administration of cationic liposome DNA aggregates. J Pharmacol Exp Ther. 1999;289: 807-815.

20. Gao J, Yu Y, Zhang Y, et al. EGFR-specific PEGylated immunoliposomes for active siRNA delivery in hepatocellular carcinoma. Biomaterials. 2012;33:270-282.

21. Bogdanov AA Jr, Martin C, Weissleder R, Brady TJ. Trapping of dextran-coated colloids in liposomes by transient binding to aminophospholipid: preparation of ferrosomes. Biochim Biophys Acta. 1994;1193: 212-218.

22. Lu J, Jeon E, Lee BS, Onyuksel H, Wang ZJ. Targeted drug delivery crossing cytoplasmic membranes of intended cells via ligand-grafted sterically stabilized liposomes. J Control Release. 2006;110:505-513.

23. Saul JM, Annapragada A, Natarajan JV, Bellamkonda RV. Controlled targeting of liposomal doxorubicin via the folate receptor in vitro. $J$ Control Release. 2003;92(1-2):49-67.

24. Kiwada H, Sato J, Yamada S, Kato Y. Feasibility of magnetic liposomes as a targeting device for drugs. Chem Pharm Bull (Tokyo). 1986;34:4253-4258.

25. Heath TD, Robertson D, Birbeck MS, Davies AJ. Covalent attachment of horseradish peroxidase to the outer surface of liposomes. Biochim Biophys Acta. 1980;599:42-62.

26. Skouras A, Mourtas S, Markoutsa E, et al. Magnetoliposomes with high USPIO entrapping efficiency, stability and magnetic properties. Nanomedicine. 2011;7:572-579.

27. Klibanov AL, Maruyama K, Beckerleg AM, Torchilin VP, Huang L. Activity of amphipathic poly(ethylene glycol) 5000 to prolong the circulation time of liposomes depends on the liposome size and is unfavorable for immunoliposome binding to target. Biochim Biophys Acta. 1991;1062:142-148.

28. Mori M, Liu D, Kumar S, Huang Z. NMR structures of anti-HIV D-peptides derived from the N-terminus of viral chemokine vMIP-II. Biochem Biophys Res Commun. 2005;335:651-658.

29. Torchilin VP. Targeted pharmaceutical nanocarriers for cancer therapy and imaging. AAPS J. 2007;9:E128-E147. 
30. Bolotin EM, Cohen R, Bar LK, et al. Ammonium sulfate gradients for efficient and stable remote loading of amphipathic weak bases into liposomes and ligandoliposomes. J Liposome Res. 1994;4: 455-479.

31. Soenen SJ, Hodenius M, De Cuyper M. Magnetoliposomes: versatile innovative nanocolloids for use in biotechnology and biomedicine. Nanomedicine (Lond). 2009;4:177-191.
32. Mulder WJ, Strijkers GJ, Griffioen AW, et al. A liposomal system for contrast-enhanced magnetic resonance imaging of molecular targets. Bioconjug Chem. 2004;15:799-806.

International Journal of Nanomedicine

\section{Publish your work in this journal}

The International Journal of Nanomedicine is an international, peerreviewed journal focusing on the application of nanotechnology in diagnostics, therapeutics, and drug delivery systems throughout the biomedical field. This journal is indexed on PubMed Central, MedLine, CAS, SciSearch $\AA$, Current Contents ${ } /$ Clinical Medicine,
Journal Citation Reports/Science Edition, EMBase, Scopus and the Elsevier Bibliographic databases. The manuscript management system is completely online and includes a very quick and fair peer-review system, which is all easy to use. Visit http://www.dovepress.com/ testimonials.php to read real quotes from published authors.

Submit your manuscript here: http://www.dovepress.com/international-journal-of-nanomedicine-journal 